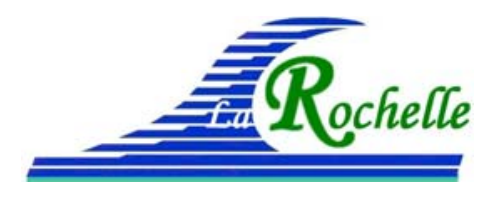

XVèmes Journées Nationales Génie Côtier - Génie Civil

La Rochelle, 29 au 31 mai 2018

DOI:10.5150/jngcgc.2018.058 C Editions Paralia CFL

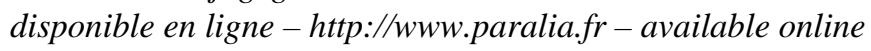

\title{
Analyse des modules tests pour les piles du viaduc de la nouvelle route du littoral (Océan Indien, île de La Réunion).
}

\section{Julien WICKEL ${ }^{1}$, Mathieu PINAULT ${ }^{1}$, Michael GRONIER ${ }^{2}$, Etienne CLAMAGIRAND ${ }^{2}$}

\author{
1. GIE Marex, 697 chemin La Surprise- 97436 Saint Leu, France. \\ math.pinault@gmail.com
}

2. ARCHITEUTHIS, 100 rue Grignan, 13001 Marseille, France.

m.gronier@architeuthis.fr; e.clamagirand@architeuthis.fr.

\section{Résumé :}

Dans le cadre du chantier de la Nouvelle Route du Littoral (NRL), en cours de réalisation sur l'île de la Réunion, la société ARCHITEUTHIS a été mandatée pour concevoir et immerger un prototype de récif artificiel pourvu de dispositifs biocompatibles destinés à équiper à terme 6 piles sur les $5,4 \mathrm{~km}$ de viaduc en mer. Ce prototype a été immergé le 30 avril 2015, à 7,5 $\mathrm{m}$ de profondeur, sur une station située à $20^{\circ} 52,996 \mathrm{~S}$ et $55^{\circ} 23,548 \mathrm{E}$, au droit du lieu-dit de la "Pointe du Gouffre".

Le prototype de récif artificiel est composé de quatre sous unités :

- Une bouée équipée de structures imitant les racines de mangrove, (module "mangrove");

- Un corps-mort d'ancrage de la bouée (module "corps-mort");

- Un cube de 1,5 m de côté équipé de panals (le récif de paroi ou "Panal" est une pièce en composite-ciment-verre constitué de plusieurs alvéoles en forme de nid d'abeille déformé. Cette architecture permet de mettre à disposition de la flore et de la faune des surfaces importantes à coloniser tout en garantissant une grande solidité structurelle) imitant les abris naturels (module "panals") ;

- Un cube de 1,5 m de côté ne présentant aucune ornementation (module "témoin").

Un suivi des modules est réalisé tous les mois durant une période d'une année suivant l'immersion des structures, l'objectif du suivi étant (i) de vérifier la bonne tenue mécanique des modules (ii) de contrôler la colonisation biologique des structures et (iii) d'en appréhender les facteurs favorisants (orientation, saisonnalité, efficacité des dispositifs biocompatibles). Seuls les modules "mangrove", "témoin" et "panals" sont suivis au cours de cette étude. Il s'agit d'améliorer le rôle de protection sur une zone dite de "recrutement" qui permet aux larves planctoniques de poissons, lors de leurs cycles saisonniers, de se rapprocher de la côte pour trouver abri et nourriture, avant de se transformer en petits poissons viables.

Mots-clés : Génie côtier, Travaux maritimes, Environnement littoral, Écosystèmes côtiers, Récifs artificiels, Eco-nurseries, Eco conception des ouvrages d'art. 


\section{Thème 4 - Ouvrages portuaires, offshore et de plaisance}
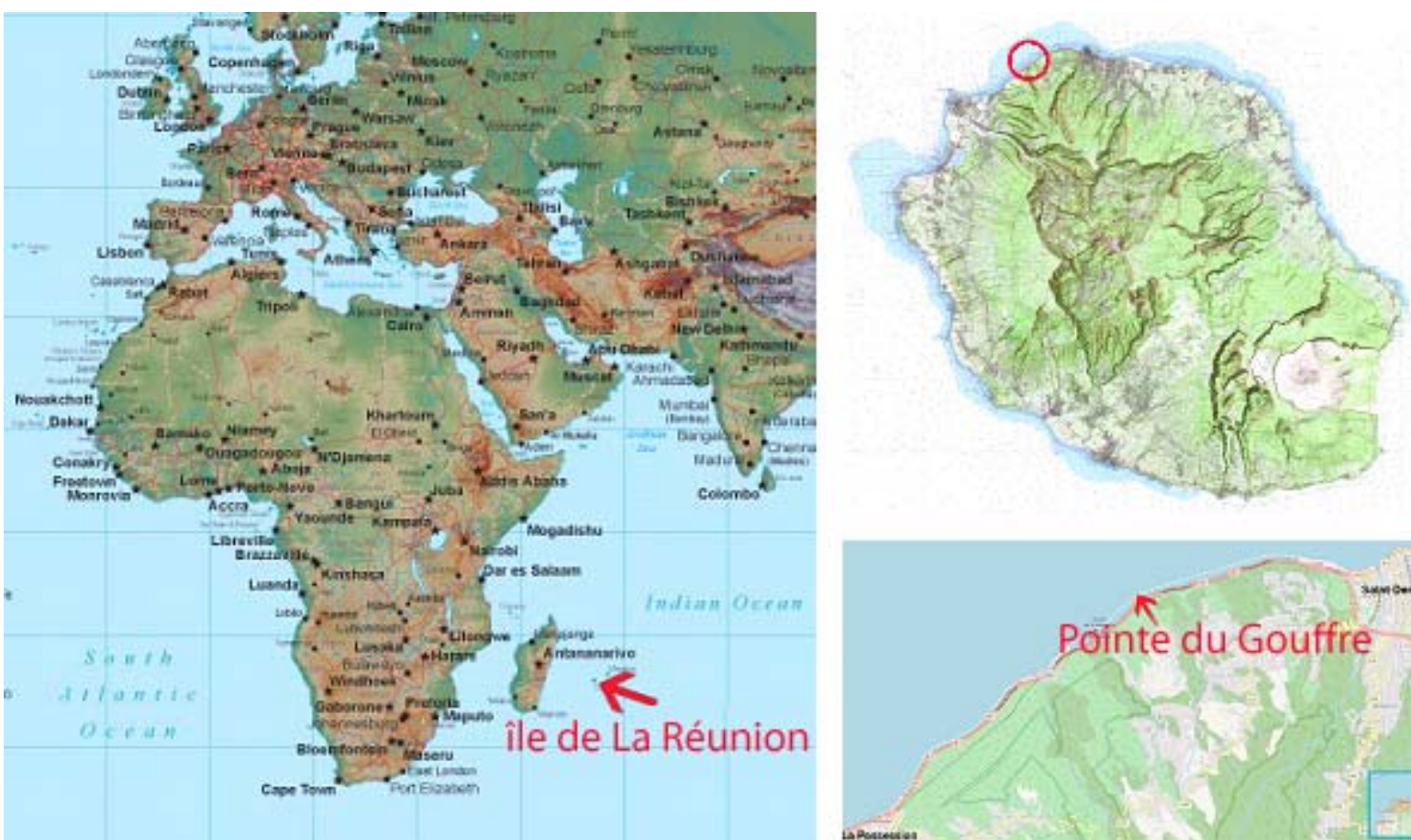

Figure 1. Cartes de situations de la Réunion, de la Nouvelle Route du Littoral et des dispositifs.

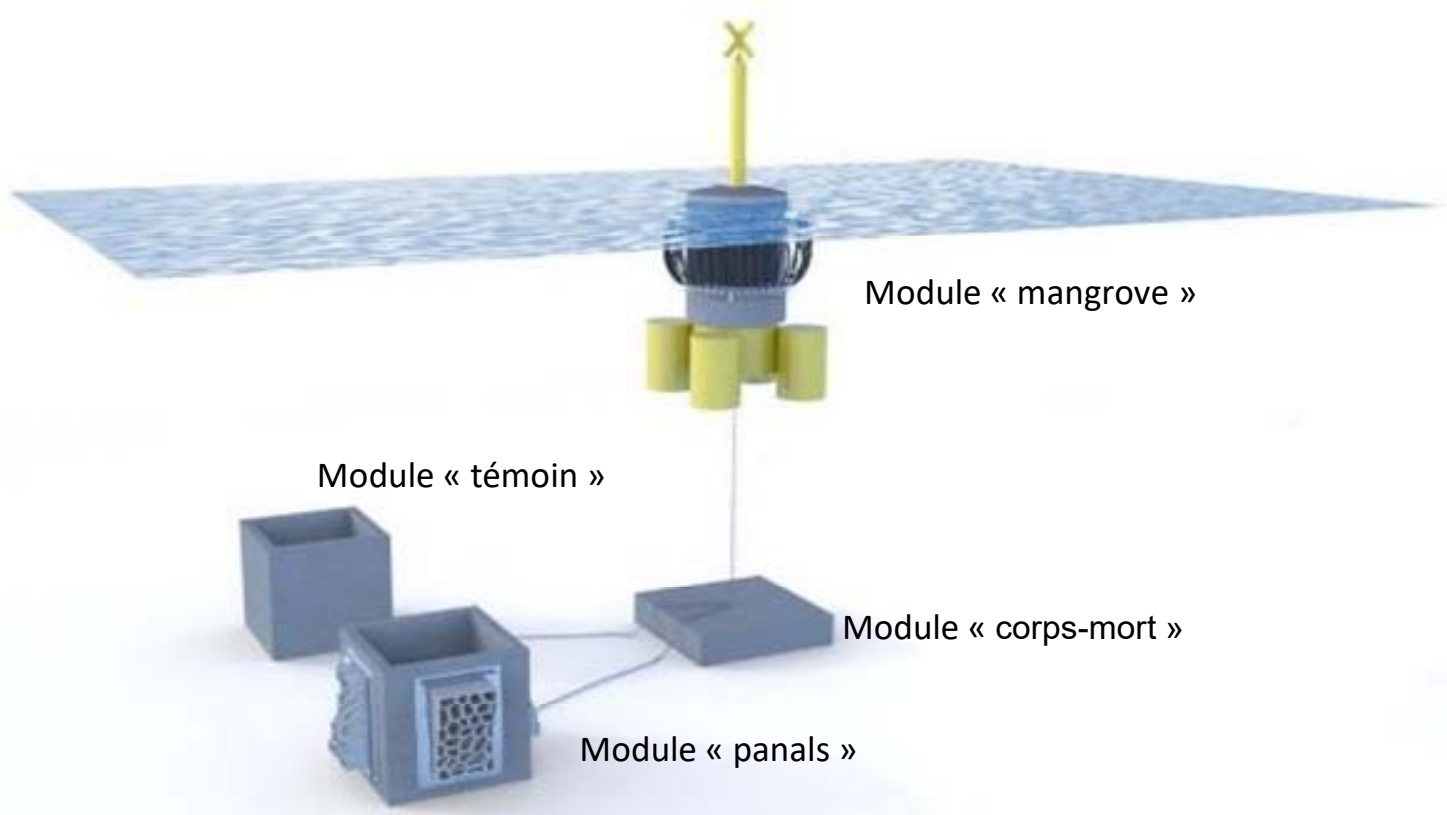

Figure 2. Schéma du dispositif test.

\section{Introduction}

L'objectif recherché dans un travail d'écoconception d'ouvrage tel que la Nouvelle Route du Littoral est de limiter et compenser les impacts résiduels du projet sur le milieu marin. Le choix d'éco-concevoir six piles de viaduc dans le secteur où est 


\section{XVèmes Journées Nationales Génie Côtier - Génie Civil \\ La Rochelle, 29 au 31 mai 2018}

présente une zone source ne sera que bénéfique pour l'exportation de la biomasse vers les habitats artificiels.

En effet, les piles situées à la Pointe du Gouffre seront ainsi positionnées au droit d'une zone source dont les peuplements sont remarquables et résilients.

De même qu'en tant que zone de maturation, la pointe du Gouffre se voit être un emplacement favorable puisque cette zone est exposée au flux larvaires. Des connectivités avec les affleurements basaltiques du large seront créées et permettront de bénéficier d'une dissémination optimale.

Compte-tenu de la sensibilité des biocénoses de substrats durs qui seront impactées par le projet et de leur rôle central dans le fonctionnement de l'écosystème, la création d'éco-modules sur six piles du viaduc en zone à enjeu écologique fort est un des éléments essentiellement pour la création de corridors biologiques.

Architeuthis, à la suite d'une consultation nationale a été retenue en se positionnant comme prestataire de service clé en main pour les problématiques d'aménagement et de restauration du littoral sous-marin. Dans cet état d'esprit, la société offre tous les services amont et aval autour de l'implantation d'un récif artificiel, ainsi que la technologie du récif elle-même. Comme les systèmes Hexapora ${ }^{\circledR}$, Fractal ${ }^{\circledR}$, Panal ${ }^{\circledR}$, l'envergure des services d'études préalables et service après-vente sont déterminés par les objectifs de chaque projet et les souhaits du client. L'offre de service autour des habitats reconstitués est entièrement adaptée aux besoins du client. Dans cette optique l'activité de l'entreprise se répartit dans une activité d'étude (préalable, de faisabilité, de biologie, d'hydrologie, etc.) et une activité d'ingénierie des produits. La planification, l'expertise, la construction et l'implantation sont également maîtrisées. Dans cette activité d'ingénierie Architeuthis travaille comme maître d'œuvre et entreprise de réalisation clefs en main. Un cahier des charges extrêmement contraint dans le domaine de l'environnement, de loin le plus précautionneux que nous ayons eu à traiter, nous a été soumis afin que nos solutions apportent des garanties de protection, voire d'amélioration du milieu marin. Les ingénieurs et les biologistes, de la société ont déterminé les deux types d'habitats artificiels qui pouvaient satisfaire à la fois les cycles naturels et la résistance structurelle. Les modules "mangrove" sont situés près de la surface de la mer (récif subsurface) et les modules "panal", en composite-ciment verre, sont fixés sur les parois verticales de la pile du viaduc à proximité du fond. Grâce à ces dispositifs uniques, La Réunion se positionne comme un territoire pionnier dans ce domaine d'avenir capital qui est celui de l'ingénierie écologique marine.

\section{Fonctionnement du cycle de vie des poissons côtiers en milieu naturel lors des périodes de reproduction}

Les poissons adultes se regroupent en pleine-eau au large (LENFANT et al., 2015). C'est là et aussi près du fond que les œufs sont émis et vont éclore, avant de se disperser en milieu pélagique au gré des courants. Une seule larve sur cent œufs survivra. En 


\section{Thème 4 - Ouvrages portuaires, offshore et de plaisance}

effet, durant la phase larvaire pélagique, la fragilité de ces nouveau-nés, la forte prédation et le déficit de nourriture sont des causes de mortalité importante pour les larves. Ensuite, ces dernières évoluent et $10 \%$ d'entre elles acquièrent le statut de postlarves. Elles se dirigent rapidement vers la côte, plus favorable à leur évolution, afin de s'y installer sur les fonds et de coloniser cet habitat en tant que nouvelles recrues. Ces quelques jours d'installation sont aussi une phase dangereuse, où le taux de mortalité est élevé (90\%). Une fois installés, ces poissons juvéniles grandissent dans un habitat favorable à leur développement où ils trouvent abris et nourriture. Puis quelques mois plus tard, ils atteignent une taille supérieure à l'ouverture de la bouche de la plupart des prédateurs, ce qui réduit sensiblement le taux de mortalité. Ils peuvent ensuite rejoindre les adultes dans leur habitat, pour continuer leur croissance avant de devenir à leur tour des adultes reproducteurs. À terme, seulement 1 poisson sur un million atteindra la phase de recrutement en habitat naturel. Le cycle de reproduction des poissons est alors très fragile. Cependant, les pressions anthropiques, bouleversent l'écosystème du milieu marin. Pour pallier à ces problèmes, la société a développé une gamme d'abris calibrés. Ces solutions permettent de limiter les pressions anthropiques, mais aussi de réhabiliter les écosystèmes endommagés.

En 2015, le Viaduc du Littoral teste les dispositifs d'écoconception des futures piles. Des modules éco conçus, sont étudiés pour favoriser la faune sous-marine et permettre au milieu marin de se reconstituer en favorisant l'éclosion d'une faune riche et la croissance des alevins dans un milieu protecteur. Six piles situées à la Pointe du Gouffre recevront des structures éco conçues adaptées. L'écoconception recoupe l'ensemble des dispositifs d'habitats artificiels intégrés aux piles du viaduc. Ces dispositifs jouent le rôle de nurserie et visent ainsi à assurer le développement et la croissance de la faune du stade de larve à celui de juvénile. Ce principe vise à intégrer directement à l'ouvrage des récifs artificiels, les piles du viaduc offrant un support adapté à la mise en place de différents modules, qui présenteront une structure similaire à des habitats naturels complexes.

Lorsque les espèces passent du stade larvaire au stade juvénile et quittent la couche superficielle de la masse d'eau, elles nécessitent des habitats de protection très particuliers, adaptés à leur installation :

Pour les espèces benthiques, des microcavités protégeant des attaques de prédateurs, ou pour les espèces démersales et pélagiques, des couloirs étroits à l'abri des attaques de prédateurs.

Pour répondre aux besoins spécifiques des espèces en présence, deux types de modules ont été réalisés : des modules de subsurface (Mangrove) et des modules de paroi (Panal). Ces deux modèles permettront aux larves et aux post-larves des poissons et crustacés d'avoir un meilleur taux de survie. Ainsi les piles du viaduc éco conçues devraient avoir un impact positif sur les populations futures de la faune sous-marine locale. 


\section{XVèmes Journées Nationales Génie Côtier - Génie Civil \\ La Rochelle, 29 au 31 mai 2018}

Pour la Nouvelle Route du Littoral, un an d'études et de mises au point a été nécessaire pour répondre aux problématiques biologiques locales et celle hors norme de la vague centennale qui peut atteindre le littoral réunionnais lors des épisodes cycloniques (résistance de $10 \mathrm{~T}$ au $\mathrm{m}^{2}$ soit l'impact d'une vague de $13 \mathrm{~m}$ de haut). Les ingénieurs et les biologistes de la société Architeuthis ont déterminé les deux types d'habitats artificiels qui pouvaient satisfaire à la fois les cycles naturels et la résistance structurelle. Les modules "Mangrove", immergés en partie haute de l'amorce du fût de pile, ces modules de subsurface auront pour but de capter les larves et post-larves à la dérive et d'offrir un micro habitat complexe et adapté aux tailles particulièrement réduites des individus au tout premier stade de développement. La forme de module type racine de mangrove dispose d'une structure en faisceaux enchevêtrés et a pour but de mimer les habitats naturels connus pour exercer cette fonctionnalité : herbier, mangrove ou coraux érigés. Le module "Mangrove" est ainsi constitué de différents tuyaux de polyéthylène disposés de manière parallèle les uns par rapport aux autres, tout autour du périmètre des piles. Le polyéthylène est un matériau inerte classé "alimentaire" (Matériaux PE Europa Safe 10 Normes (UNI) EN 12201 ISO 4427). Pour compléter ce dispositif, un géotextile est placé entre le béton de l'ouvrage et les tuyaux.

Les espèces ciblées sont les larves et post-larves d'espèces démersales récifales : demoiselles, capucins, poissons-chirurgiens, labres, vivaneaux, poissons-papillons.

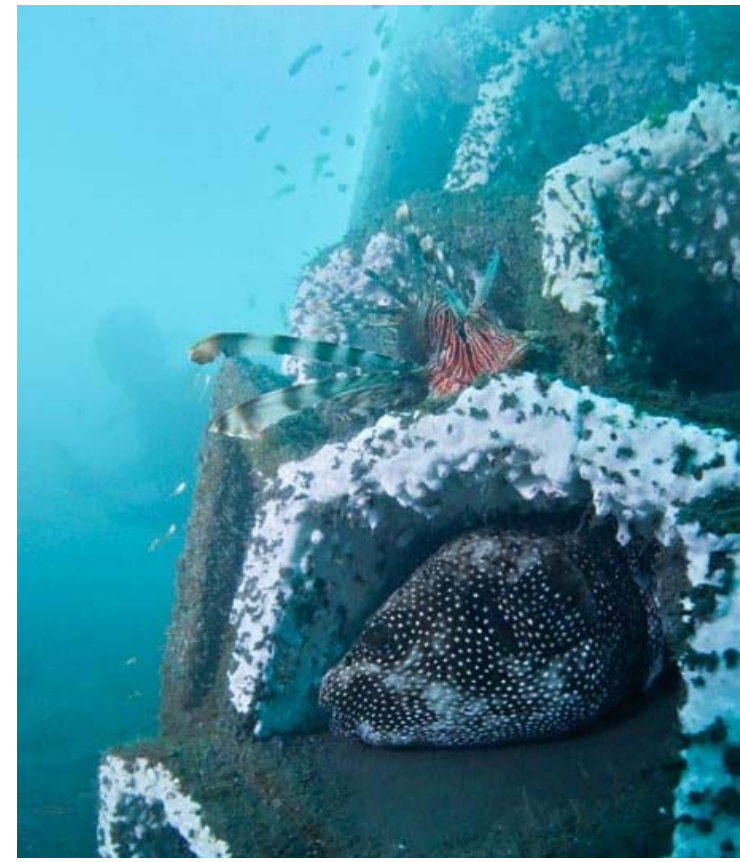

Figure 3. Panal test après 4 mois d'immersion.

Les modules pariétaux "Panal" placés en partie basse du fût de pile, assurent la transition entre les modules de subsurface et les fonds. Ces modules consistent à 


\section{Thème 4 - Ouvrages portuaires, offshore et de plaisance}

complexifier les parois lisses des piles du viaduc, en offrant des supports à même de favoriser le développement d'une faune et d'une flore fixée riche (figure 3), en ciblant les espèces coralliennes. La forme du module consiste en un "nid d'abeille" déformé, en vue d'obtenir un réseau irrégulier sous forme de trame hexagonale. Ces récifs en béton sont réalisés en Composite Ciment Fibre de Verre (CCV). Ces matériaux "éco ciments" sont composés de produits inertes ou rendus inoffensifs pour les milieux naturels. Les espèces coralliennes ciblées sont celles existantes sur le site : porites, pavones, millepores, pocillopores, acropores.

Les objectifs de ces dispositifs ne sont pas d'ordres halieutiques ou commerciaux, mais permettent de reconstituer un continuum écologique, entre la Pointe du Gouffre et les substrats rocheux profonds, qui seraient perturbés par l'implantation des piles.

Depuis avril 2015 les équipes du viaduc et d'Architeuthis ont immergé un module test au niveau de la pointe du Gouffre (zone écologique sensible).

Ce dispositif est suivi mensuellement par les plongeurs, mandatés par la société, afin de relever les différentes espèces sous-marines en présence et ils effectuent un relevé photographique détaillé. Ce suivi permet une appréhension fine de l'évolution biologique du milieu et de la résistance mécanique des modules.

\section{Résultats}

Un total de 40 espèces et 17 familles a été recensé au cours des neuf campagnes de suivi sur les 3 modules étudiés (figure 4). Le module "mangrove" accueillait ainsi 8 espèces et 6 familles, le module "témoin", 9 espèces et 7 familles et le module "panals", 32 espèces et 16 familles. La richesse spécifique bien supérieure observée sur le module "panals" est en faveur d'un fonctionnement écologique significatif des ornementations en nid d'abeille. Cet effet est dû à la création de nombreux petits habitats au sein des alvéoles, absents des deux autres modules et offrant de nouvelles loges potentielles à de nombreuses espèces aux exigences écologiques différentes (cavernicole, cryptique, territoriale, etc.). Ces cavités de tailles et de profondeurs différentes de l'ordre du décimétrique et $\mathrm{du}$ centimétrique, seront naturellement complétées par des anfractuosités millimétriques lors de la colonisation corallienne.

Les densités moyennes mesurées présentent de très nettes différences entre modules. Le module "mangrove" présente les plus faibles densités de poissons avec en moyenne 44 \pm 20 (Erreur type) indiv.100 $\mathrm{m}^{-2}$. Vient ensuite le module "témoin", avec $496 \pm 237$ indiv. $100 \mathrm{~m}^{-2}$. Les valeurs maximales sont mesurées sur le module "panals" avec $2814 \pm$ 730 indiv. $100 \mathrm{~m}^{-2}$.

Les biomasses moyennes présentent également des différences importantes entre modules avec $52 \pm 48 \mathrm{~g} \mathrm{~m}^{-2}$ pour le module "mangrove", $48 \pm 23 \mathrm{~g} \mathrm{~m}^{-2}$ pour le module "témoin" et $315 \pm 86 \mathrm{~g} \mathrm{~m}^{-2}$ pour le module "panals". On observe ainsi des valeurs globalement plus élevées mesurées sur le module "panals". En revanche, si les densités sont bien supérieures sur le module "témoin" que sur le module "mangrove", les valeurs 


\section{XVèmes Journées Nationales Génie Côtier - Génie Civil \\ La Rochelle, 29 au 31 mai 2018}

de biomasses de ces deux modules sont très similaires. Ce paradoxe est probablement dû au fait que le module "témoin" est colonisé par de nombreux très petits poissons tandis que le module "mangrove" accueille des peuplements plus épars, mais constitués d'individus de plus grande taille.

\begin{tabular}{|c|c|c|}
\hline FAMILLE & Espèce & Nom vernaculaire \\
\hline \multirow{2}{*}{ ACANTHURIDAE } & Acanthurus guttatus & Chirurgien pintade \\
\hline & Acanthurus mata & Chirurgien pâle \\
\hline \multirow[t]{4}{*}{ APOGONIDAE } & Ostorhynchus sp1 & Apogon doré \\
\hline & Ostorhynchus sp2 & \\
\hline & Ostorhynchus sp3 & \\
\hline & Ostorhynchus sp4 & \\
\hline BALISTIDAE & Sufflamen fraenatum & Baliste à muselière \\
\hline \multirow[t]{2}{*}{ BLENNIIDAE } & Aspidontus dussumieri & Blennie lance \\
\hline & Plagiotremus tapeinosoma & Blennie à dents acérées \\
\hline CAESIONIDAE & Pterocaesio marri & Fusilier de Marr \\
\hline \multirow[t]{2}{*}{ CHAETODONTIDEA } & Chaetodon vagabundus & papillon vagabond \\
\hline & Heniochus acuminatus & poisson cocher \\
\hline \multirow[t]{2}{*}{ CIRRHITIDAE } & Cirrhitchys guichenoti & Epervier des Mascareignes \\
\hline & Cirrhitchys pinnulatus & Epervier de corail \\
\hline DIODONTIDAE & Chilomycterus reticulatus & Diodon à nageoires tachetées \\
\hline \multirow[t]{5}{*}{ LABRIDAE } & Cheilinus trilobatus & Labre à queue triple \\
\hline & Coris caudimacula & Girelle à tache caudale \\
\hline & Gomphosus caeruleus & Labre oiseau indien \\
\hline & Halichores cosmetus & Labre décoré \\
\hline & Labroides diminiatus & Labre nettoyeur commun \\
\hline \multirow[t]{3}{*}{ LUTJANIDAE } & Lutjanus fulvus & Vivaneau à queue noire \\
\hline & Lutjanus gibbus & Vivaneau pagaie \\
\hline & Lutjanus Kasmira & Vivaneau à raies bleues \\
\hline MULLIDAE & Upeneus tragula & Capucin souris à bande sombre \\
\hline PLOTOSIDAE & Plotosus lineatus & Poisson chat rayé \\
\hline \multirow[t]{4}{*}{ POMACENTRIDAE } & Abudefduf vaigiensis & Poisson bagnard \\
\hline & Chromis lepidolepis & Chromis écailleux \\
\hline & Chromis nigrura & Chromis de l'océan indien \\
\hline & Pomacentrus caeruleus & Demoiselle bleue \\
\hline \multirow[t]{2}{*}{ SCORPANIDAE } & Pterois miles & Rascasse volante \\
\hline & Scorpaenopsis sp. & Poisson scorpion \\
\hline \multirow[t]{3}{*}{ SERRANIDAE } & Epinephelus hexagonatus & Mérou mélifère \\
\hline & Epinephelus oceanicus & Rougette \\
\hline & Pseudanthias squamipinnis & Anthias commun \\
\hline \multirow[t]{2}{*}{ TETRAODONTIDAE } & Arothron hispidus & Poisson ballon à taches blanches \\
\hline & Arothron meleagris & Poisson ballon pintade \\
\hline ZANCLIDAE & Zanclus cornutus & Idole maure \\
\hline
\end{tabular}

Figure 4. Liste des espèces de poissons recensées au cours des campagnes $n^{\circ} 1$ à 9 . 


\section{Thème 4 - Ouvrages portuaires, offshore et de plaisance}

\section{Conclusions}

\subsection{Les modules Mangroves}

Le dispositif de la bouée support mis à disposition par le groupement bénéficiaire du marché de la NRL (Vinci Construction Grands Projets - Bouygues Travaux Publics Dodin Campenon Bernard - Demathieu Bard) n'a pas supporté les conditions de mer violentes du site. Il a été décidé par le Maître d'Ouvrage (Région Réunion) d'arrêter l'expérience, après un premier naufrage de la bouée, malgré les premiers résultats prometteurs, la bouée a été rapatriée à terre. Le test d'un an n'ayant pu avoir lieu, il a été décidé de ne pas installer le récif "Mangrove" sur les piles.

\subsection{Les Panals}

L'expérimentation du dispositif test a prouvé son efficacité. La mise en place des modules Panal sur les piles a donc été décidée (figures 7 et 8 ). Le rôle dédié de structure de connexion étant avéré, doublé d'un rôle d'une nouvelle zone trophique. Depuis ces piles ont été posée et il s'avère que celle-ci on été très rapidement colonisées (figures 5 et 6).

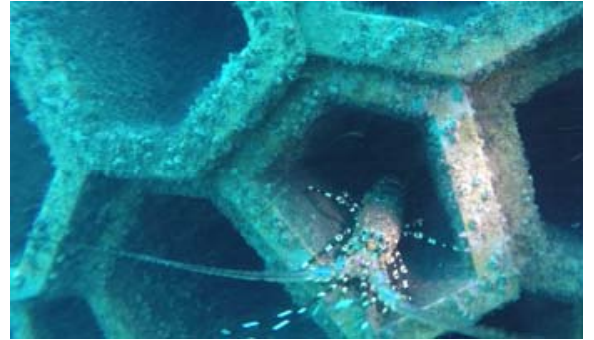

Figure 5. Après trois mois d'immersion langouste rouge (palinurus longipedes).

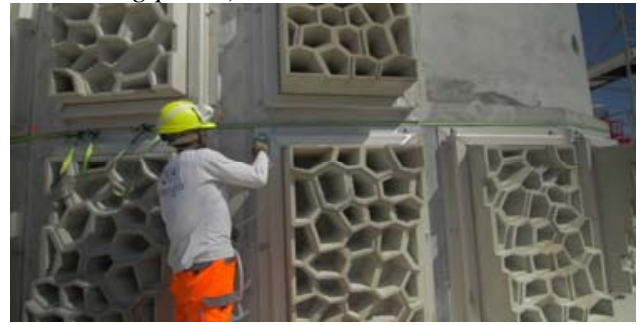

Figure 7. Mise en place des Panals.

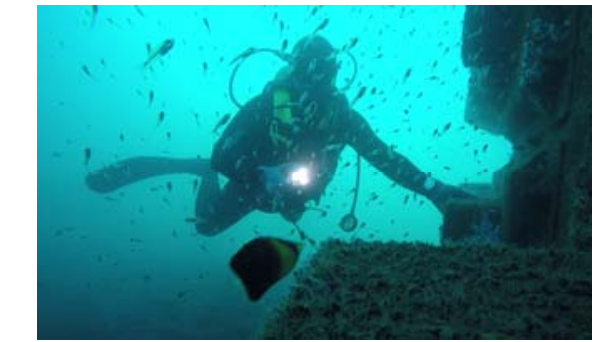

Figure 6. Post-larves colonisant les Panals (trois mois d'immersion).

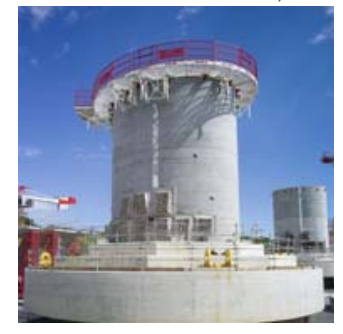

Figure 8. Vingt modules Panals sont scellés sur chacune des six piles de viaduc.

\section{Référence bibliographique}

LENFANT P., GUDEFIN A., FONBONNE S., LECAILLON G., ARONSON J., BLIN E., LOURIE S.M., BOISSERY P., LOEUILLARD J.-L., PALMARO A., HERROUIN G., PERSON J. (2015). Restauration écologique des nurseries des petits fonds côtiers de Méditerranée. Orientations et principes, Rapport d'étude, 95 p. 